\title{
OS BENEFÍCIOS DA INICIAÇÃO CIENTÍFICA PARA O DESENVOLVIMENTO DOS ALUNOS DE GRADUAÇÃO EM ENGENHARIA SEGUNDO EX-MEMBROS
}

DOI: 10.37702/2175-957X.COBENGE.2021.3609

Paulo Henrique Liberato da Silva - paulo.liberato@aluno.ufop.edu.br Universidade Federal de Ouro Preto

Rua Arthur Versiani Machado 590

35400-000 - Ouro Preto - MG

Thiago Duarte Figueiredo - thiagoduarte1926@gmail.com

Universidade Federal de Ouro Preto

Rua Pedro Alexandrino Rufino 76

35400-000 - OURO PRETO - MG

Caio Cesar Gonzaga Alves - caioalvesbnk@gmail.com

Universidade Federal de Ouro Preto

Luis pereira de souza 88

36204-422 - Barbacena - MG

João Octávio Carvalho Sáez Oliveira - joao.saez@aluno.ufop.edu.br Universidade Federal de Ouro Preto

Rua Conselheiro Quintiliano 350

35400-000 - Ouro Preto - MG

Carlos Alberto Pereira - pereiraufop@gmail.com

Universidade Federal de Ouro Preto

Rua João XXIII 240

35400-000 - Ouro Preto - MG

Resumo: A Iniciação Científica (IC) representa uma das mais importantes atividades para o estudante de graduação. Muitas vezes responsável pelo primeiro contato do aluno à pesquisa na área acadêmica, a IC é ferramenta notável no desenvolvimento do estudante de engenharia, dada a participação em pesquisas importantes para sua área de atuação, a necessidade de estudos aprofundados sobre o tema e a possibilidade de trabalho em conjunto com empresas e 
profissionais atuantes, exigindo empenho, maturidade e constante evolução. Com isso, o artigo visa analisar qual o ponto de vista de ex-alunos de IC em relação à importância da participação nas atividades de iniciação cientifica durante a graduação, tanto na evolução técnica quanto pessoal dos mesmos. Para isso, foi realizado um questionário aos ex-membros de IC do curso de engenharia de minas da Universidade Federal de Ouro Preto, arguindo-os sobre o quão importante foi a participação em projetos IC para suas conquistas pessoais e profissionais. As respostas obtidas permitiram constatar a tendência de entrada dos alunos aos projetos nos períodos iniciais do curso e após o fim do ciclo básico, com tempo médio de permanência de 2 anos, além de, segundo os ex-membros, proporcionar grande conhecimento técnico aos participantes, além de possibilitar o desenvolvimento de características como a organização, a boa relação com os companheiros e colegas de trabalho e a maturidade, conhecer um pouco mais o ambiente de pesquisa acadêmica e de atuação industrial e, assim, se preparar melhor e com maior potencial de sucesso.

Palavras-chave: Iniciação científica. Pesquisa acadêmica. Atividades extracurriculares. 


\section{OS BENEFÍCIOS DA INICIAÇÃO CIENTÍFICA PARA O DESENVOLVIMENTO DOS ALUNOS DE GRADUAÇÃO EM ENGENHARIA SEGUNDO EX-MEMBROS}

\section{INTRODUÇÃO}

A Iniciação Científica, como o próprio nome sugere, consiste em uma atividade de introdução do aluno ao ambiente da pesquisa e desenvolvimento na sua área de atuação, sendo muitas vezes a primeira oportunidade de contato do estudante a conhecimentos específicos da sua área de atuação e de produção acadêmica (CALAZANS, 2002). Com os primeiros registros de projetos de IC no Brasil remontados à metade do século XX, a realização das atividades é subsidiada por programas de fomento à pesquisa como $\mathrm{CNPq}$, consistindo em ferramentas importantes de incentivo a iniciação cientifica por meio de apoio financeiro, muitas vezes fundamental para a manutenção do aluno na graduação (SILVA, 2012).

Ao ingressar em cursos de engenharia o aluno é apresentado inicialmente ao ciclo básico, abordando apenas conteúdos comuns a todos os cursos da área, como as disciplinas de cálculo, física e química, o que muitas vezes desmotiva o aluno por não ter contato com temas específicos à área de atuação escolhida, potencializando inclusive a evasão nos períodos iniciais (ASSUNÇÃO et al., 2017). Dessa forma, a IC se torna uma ponte importante para inserir conceitos específicos no desenvolvimento do aluno nos períodos iniciais, motivando-o e reduzindo as chances de evasão, além de prepará-lo para os conteúdos que serão abordados ao longo do curso e aprimorar a capacidade de pesquisa e escrita científica.

Além do desenvolvimento técnico, a iniciação científica proporciona também um desenvolvimento pessoal significativo ao aluno. Segundo Maldonado e Paiva (1999, p. 156) a IC permite ao aluno desenvolver sua autonomia e independência, fatores diferenciais para a vida e a carreira profissional dos estudantes. PINHO (2017) afirma que a iniciação científica tem como principais benefícios, além da significativa evolução técnica do aluno, a interação do mesmo em diferentes ambientes, como grupos de pesquisas e congressos, permitindo um contato mais próximo com profissionais da área e outros nomes relevantes no cenário acadêmico e industrial

A introdução do aluno na iniciação científica, representa uma fonte de conhecimento complementar à graduação e aprimora suas competências e desenvolve habilidades técnicas e humanas importantes para os desafios que a vida profissional proporciona, tornando-os mais aptos a se tornarem engenheiros de sucesso (NETO, SOUZA e MILAK, 2019). Seguindo tais preceitos o curso de engenharia de minas da Universidade Federal de Ouro Preto tem como uma de suas prioridades a inclusão de cada vez mais alunos em projetos de Iniciação Científica, com seus docentes sempre em busca de novos projetos a fim de garantir o desenvolvimento técnico de seus alunos assim como a formação de profissionais mais preparados para assumir os desafios da engenharia após a graduação.

Sabendo da importância da Iniciação Científica para o desenvolvimento do aluno e o aprimoramento complementar à graduação, o artigo visa analisar qual foi a importância da IC na evolução técnica e pessoal dos ex-alunos de engenharia de minas da Universidade Federal de Ouro Preto que foram membros de projetos de iniciação ao longo dos últimos anos. A partir de consultas aos próprios ex-alunos será possível avaliar do ponto de vista dos mesmos o quão importante foi o período de IC para suas conquistas pessoais e profissionais. 


\section{METODOLOGIA}

A fim de mensurar o quanto a iniciação científica agregou na vida acadêmica, pessoal e profissional de ex-alunos da Universidade Federal de Ouro Preto, foi elaborado um questionário, com perguntas de múltipla escolha referentes ao período letivo de entrada e tempo de permanência em projetos de IC. De forma semelhante, foram realizadas questões discursivas acerca da importância da participação em projetos de iniciação científica para a carreira e o desenvolvimento pessoal do aluno, permitindo aos consultados expor de forma livre os principais pontos de transformação promovidos pela atuação na IC. O questionário foi encaminhado aos ex-alunos da UFOP que foram membros de projetos de iniciação científica relacionados ao curso de engenharia de minas.

Após dez dias de coleta de dados, a análise das respostas foi feita de forma estatística e com o auxílio do método do Discurso do Sujeito Coletivo (DSC), o qual consiste em agrupar respostas semelhantes de forma representativa, transformando as diversas declarações obtidas em um único discurso que compreenda a opinião geral dos consultados.

\section{AVALIAÇÃO DAS RESPOSTAS}

Após a consulta aos ex-alunos e antigos membros de projetos de Iniciação científica na UFOP, as respostas dos mesmos foram analisadas. Inicialmente buscou-se avaliar o período de entrada dos entrevistados em seu primeiro projeto de IC, cujas respostas estão representadas graficamente pela Figura 1.

Figura 1 - Período de entrada dos ex-alunos na Iniciação Científica.

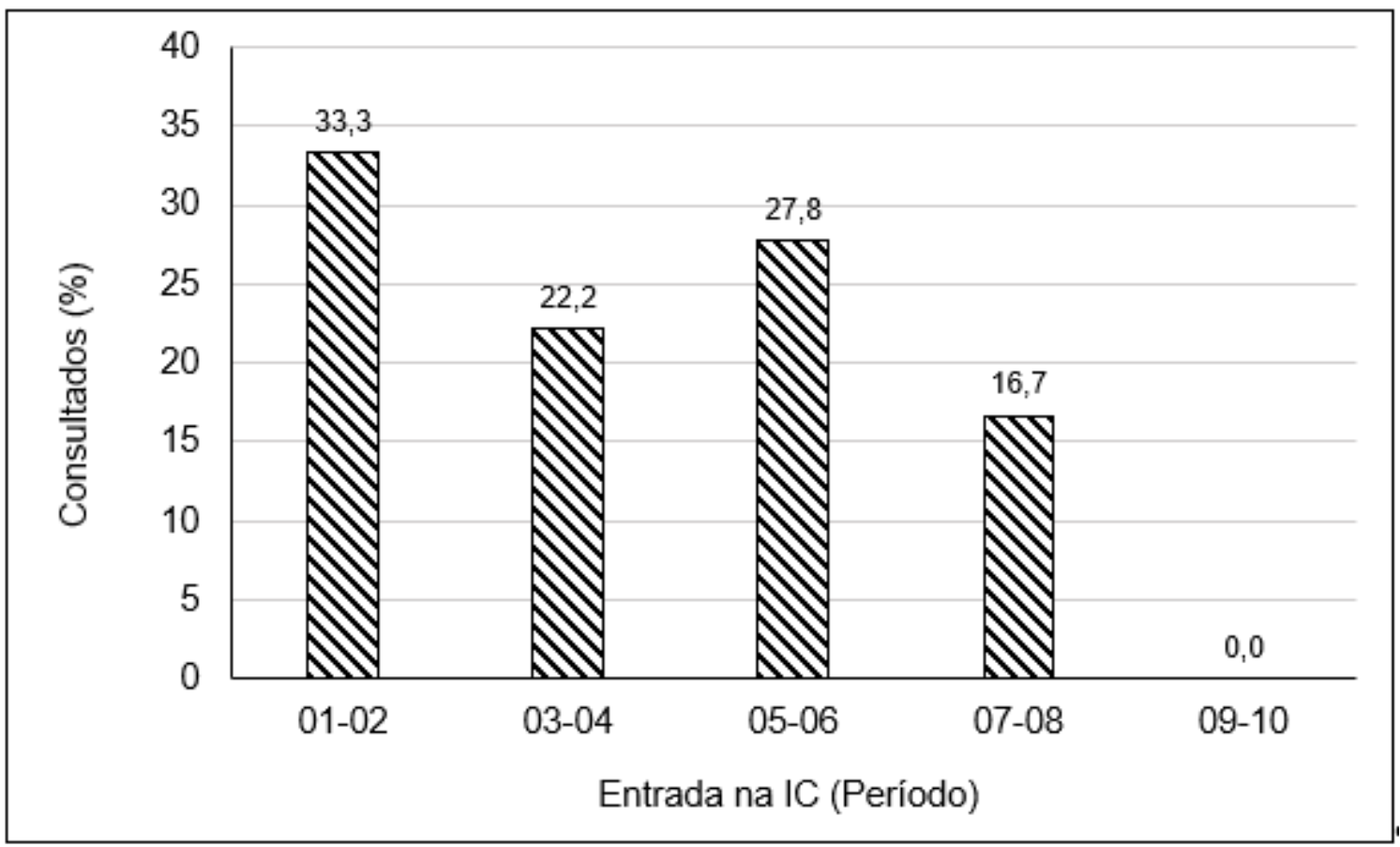

Fonte: Autoria própria

Verifica-se que a maior parte dos consultados tiveram seu primeiro contato com a iniciação científica nos primeiros dois períodos da graduação, tendência justificada pelo interesse inicial dos alunos em aprofundar seus conhecimentos técnicos ao longo do ciclo 
básico, além da maior disponibilidade de tempo para a pesquisa e do incentivo por parte dos professores orientadores, que durante as disciplinas introdutórias da graduação não medem esforços em angariar novos membros para fomento da pesquisa e produção acadêmica na instituição.

Nota-se também uma parte significante de alunos cuja participação em projetos de IC se deu no período intermediário do curso, entre o $5^{\circ}$ e 6으 período, correspondente ao fim do ciclo básico e início das disciplinas específicas do curso de engenharia. Presume-se que esta parcela de alunos, ao ser introduzida oficialmente às disciplinas específicas buscou a iniciação científica como forma de aprimorar os conteúdos abordados durante as aulas, além de se sentirem mais preparados com os temas estudados e, com isso, mais confiantes para assumirem um projeto de iniciação científica. Vale ressaltar a baixa adesão de alunos nos períodos finais do curso, justificado pela falta de disponibilidade para dedicação à projetos de IC, visto que nesse ponto do curso surgem novas prioridades como a busca por estágios e a produção do trabalho de conclusão de curso.

A Figura 2 apresenta a duração média da participação dos alunos consultados em seus respectivos projetos de iniciação científica. Destaca-se que a maior parte dos alunos permaneceu por cerca de dois anos como membros dos projetos de IC.

Figura 2 - Tempo de permanência dos ex-alunos em projetos de Iniciação Científica.

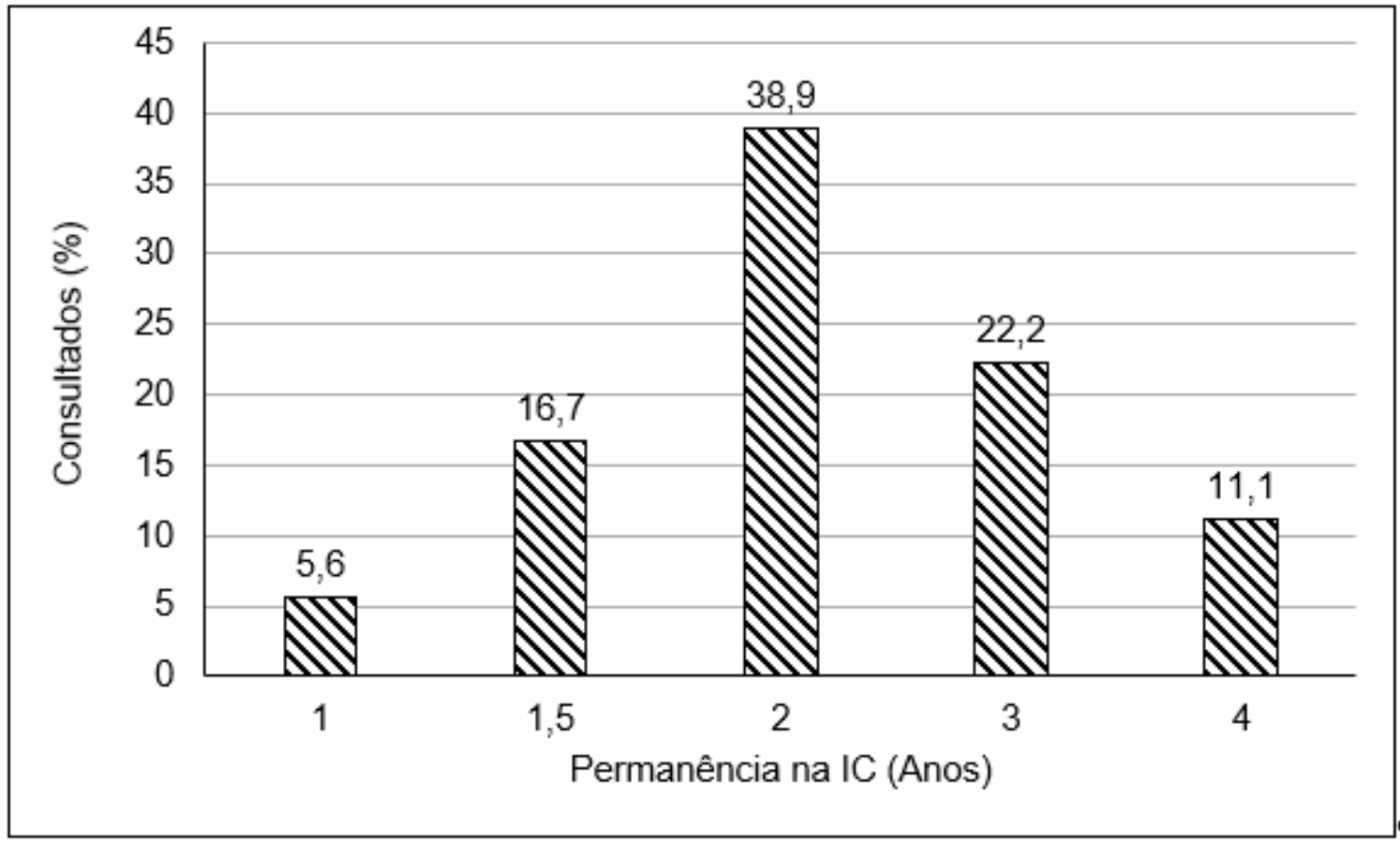

Fonte: Autoria própria

Esse período coincide com a duração dos projetos de pós graduação da universidade, onde muitos alunos da graduação são inseridos como bolsistas de pesquisas de mestrado, aprofundando seus conhecimentos prático se auxiliando os pós graduandos. Dessa forma, os projetos de mestrado são realizados com maior eficiência, dada a participação dos alunos da graduação, além de se tornarem projetos de IC com forte valor acadêmico, já que consistem, evidentemente, em pesquisas relevantes e com intensa carga de conhecimento agregado aos alunos. 
Após a análise estatística das perguntas acima, houve o agrupamento pelo DSC e discussão das respostas, levando à algumas reflexões acerca do que foi relatado pelos participantes.

Quanto à melhora e desempenho de alunos que participaram de IC durante a graduação, pôde-se analisar uma melhora em pontos como escrita, visto que é uma atividade que exige a elaboração de relatórios para apresentar os resultados e pesquisa para o desenvolvimento do projeto, o que também leva a uma melhor compreensão dos conteúdos teóricos estudados nas disciplinas, tendo a possibilidade de colocá-los em prática. Outro aspecto relevante é a autonomia e a organização que são desenvolvidas, dado a liberdade para montar horários e necessidade de cumprir metas e prazos, o que.

Contudo, além do desenvolvimento acadêmico, há também um grande desenvolvimento no âmbito profissional, pois é uma responsabilidade que ao ser assumida, se assemelha com o que será enfrentado num futuro emprego. Dentre os principais pontos citados nesse desenvolvimento, pode-se levar em consideração o direcionamento que o aluno tem para a escolha da área na qual irá atuar, visto que tem um contato tanto prático, quanto com informações e pessoas que deixam claras como será a rotina em uma empresa, posteriormente. Com essa rede instaurada durante a execução de determinados projetos, uma porta que se abre é o contato com gerentes de empresas, facilitando, de certa forma, conseguir um emprego ou estágio. E a forma de se relacionar com engenheiros já formados também pode ser trabalhada durante a iniciação, pois conversando entre eles, há uma melhora na oratória, o que é um fator muitas vezes decisivo ao se candidatar a uma vaga para ser líder de uma equipe.

Nota-se também um grande impacto no desenvolvimento pessoal, no qual devido à grande dimensão das atividades a serem desenvolvidas exige um amadurecimento e responsabilidade por parte dos, então, docentes, aliado ao empenho e desenvolvimento do trabalho em equipe, aprendendo a respeitar opiniões, impor as próprias sem ofensas e contornar problemas da melhor forma possível.

\section{CONCLUSÕES}

Por todo o exposto no presente trabalho, nota-se que, por meio da coleta de dados obtidos através de questionários respondidos por ex-alunos da Universidade Federal de Ouro Preto, observa-se o quão importante é a Iniciação Científica no desenvolvimento pessoal, acadêmico e profissional do aluno. Foi constatada uma tendência de entrada dos alunos aos projetos nos períodos iniciais do curso e após o fim do ciclo básico e início das disciplinas específicas, sendo ferramenta importante de facilitação do entendimento e prática de conceitos abordados em certas disciplinas, além de direcionar o aluno para a área que mais se identifica.

Foi observado também o tempo médio de 2 anos de permanência em projetos de iniciação científica, o que permite ao estudante adquirir um conhecimento agregado de grande valia que o ajuda no decorrer do curso, permite realizar conexões com profissionais da área e maior contato com o ambiente acadêmico e industrial da engenharia. Além disso, o discurso dos ex-alunos evidencia o grande desenvolvimento de características como a organização, a boa relação com os companheiros e colegas de trabalho e a maturidade, vista a responsabilidade que lhe é empregada. Sendo assim, a Iniciação Científica faz com que o aluno se aprimore, tornando-se um profissional com grandes chances de destaque ao longo da carreira.

\section{Agradecimentos}


Os autores agradecem a Universidade Federal de Ouro Preto pelo apoio à realização desse trabalho.

\title{
REFERÊNCIAS
}

ASSUNÇÃO, Márcio L. M. et al. A Importância da Iniciação Científica no Início do Curso de Engenharia de Controle e Automação para a Formação de Futuros Engenheiros. In: XLV Congresso Brasileiro de Educação em Engenharia, 2017, Joinville. Anais. Joinville. Disponível em:http://www.abenge.org.br/sis artigo doi.php?e=COBENGE\&a=17\&c=661. Acesso em 12 mar.2021.

CALAZANS, Maria Julieta Costa. Iniciação Científica: construindo o pensamento crítico. 2002. Ed, São Paulo: Cortez, 2002.

MALDONADO, Luciana A.; PAIVA, Edil Vasconcelos. A Iniciação Científica na Graduação em Nutrição: Possibilidades e Contribuições para a Formação Profissional. In CALAZANS, Julieta (Org), Iniciação Científica: construindo o pensamento crítico. São Paulo: Cortez, 1999. p. 156.

NETO, João M.; SOUZA, Diego L. L.; MILAK, Pâmela C. Impacto da Iniciação Científica na Engenharia Mecatrônica. In: XLVII Congresso Brasileiro de Educação em Engenharia, 2019, Fortaleza. Anais. Fortaleza. Disponível

em:http://www.abenge.org.br/sis artigo doi.php?e=COBENGE\&a=19\&c=2510. Acesso em 12 mar.2021.

PINHO, M. J. Ciência e Ensino: contribuições da iniciação científica na educação superior. Revista Avaliação, Campinas, v. 22, n. 3, p. 658-675, 2017.

SILVA, L. F. F. Iniciação Científica: contexto e aspectos práticos. Revista de Medicina, São Paulo. v. 91, n. 2, p. 128-36, 2012.

\section{THE BENEFITS OF UNDERGRADUATION RESEARCH FOR THE DEVELOPMENT OF GRADUATE STUDENTS IN ENGINEERING ACCORDING TO FORMER MEMBERS}

\begin{abstract}
Undergraduate research (UR) represents one of the most important activities for undergraduate students. Often responsible for the student's first contact with research in the academic area, UR is a remarkable tool in the development of the engineering student, promoting participation in important research for their area of expertise and the possibility of working together with consolidated companies and renowned professionals, requiring indepth studies on the subject, in addition to commitment, maturity and constant evolution. Thus, this article aims to analyze the point of view of UR former members in relation to the importance of participation in undergraduate research activities during graduation in their technical and personal evolution. For this, a questionnaire was conducted to the UR former members of the mining engineering course of the Federal University of Ouro Preto, arguing them about how important was the participation in UR projects for their personal and professional achievements. The answers obtained allowed to verify the tendency of students to enter the projects in the initial periods of the course and after the end of the basic cycle, with an average length of stay of 2 years. In addition, according to the former members, the
\end{abstract}


UR was responsible to provide great technical knowledge, besides enabling the development of characteristics such as organization, good relationship with co-workers, oratory and maturity, making students better prepared and, consequently, with greater potential for success.

Keywords: Undergraduate research. Academic Research. Extracurricular Activities. 